\title{
Classifying mild traumatic brain injuries with functional network analysis
}

\author{
F. Anthony San Lucas ${ }^{1}$, John Redell ${ }^{2}$, Dash Pramod ${ }^{2,3}$ and Yin Liư 2,3,4* \\ From The International Conference on Intelligent Biology and Medicine (ICIBM) 2018 \\ Los Angeles, CA, USA. 10-12 June 2018
}

\begin{abstract}
Background: Traumatic brain injury (TBI) represents a critical health problem of which timely diagnosis and treatment remain challenging. TBI is a result of an external force damaging brain tissue, accompanied by delayed pathogenic events which aggravate the injury. Molecular responses to different mild TBI subtypes have not been well characterized. TBI subtype classification is an important step towards the development and application of novel treatments. The computational systems biology approach is proved to be a promising tool in biomarker discovery for central nervous system injury.

Results: In this study, we have performed a network-based analysis on gene expression profiles to identify functional gene subnetworks. The gene expression profiles were obtained from two experimental models of injury in rats: the controlled cortical impact and the fluid percussion injury. Our method integrates protein interaction information with gene expression profiles to identify subnetworks of genes as biomarkers. We have demonstrated that the selected gene subnetworks are more accurate to classify the heterogeneous responses to different injury models, compared to conventional analysis using individual marker genes selected without network information.

Conclusions: The systems approach can lead to a better understanding of the underlying complexities of the molecular responses after TBI and the identified subnetworks will have important prognostic functions for patients who sustain mild TBIs.
\end{abstract}

Keywords: mTBI subtype classification, Biomarkers, Weighted protein interaction network, Subnetwork modularity, Gene ontology annotation

\section{Background}

Traumatic brain injury (TBI) results from an external force causing immediate damage to brain tissue, followed by secondary pathogenic events which ultimately give rise to neurodegeneration. Dependent on the context of the primary injury, different cell responses are initiated, which can exacerbate the injury to varying degrees. Cell death resulting from the initial impact on the brain tissue is irreversible, so treatments normally focus on minimizing the secondary injury that is due to these

\footnotetext{
* Correspondence: Yin.Liu@uth.tmc.edu

${ }^{2}$ Department of Neurobiology and Anatomy, University of Texas Health

Science Center at Houston, 6431 Fannin Street, Houston, TX, USA

${ }^{3}$ University of Texas Graduate School of Biomedical Science, 6767 Bertner

Avenue, Houston, TX, USA

Full list of author information is available at the end of the article
}

cell responses [1]. To date, these secondary injury responses have been poorly characterized, leaving molecular classification of TBI difficult [2, 3]. TBI remains a leading cause of death and disability in the industrialized countries and represents a growing health problem [4]. Thus, even a modest improvement in patient outcome could have significant public health benefits $[5,6]$. It is estimated that at least $25 \%$ of patients experiencing a mild TBI (mTBI) do not seek hospital care [7]. Among these mTBI patients, some of the post-concussive symptoms have been reported to remain up to one year or more and can significantly affect the long-term morbidities [8]. It has been shown that concussive force can elicit physical and structural changes in the brain. These changes can be focal or diffuse through the brain [9]. Therefore, identification of both common and 
pathology-specific molecular mechanisms underlying different types of injuries may aid in identification of targets for effective TBI treatments. We have utilized two common experimental models of injury in this study: the mild controlled cortical impact (mCCI) model that causes a focal injury, and the mild fluid percussion injury (mFPI) model that causes a more diffuse brain injury. Both injury models qualitatively recapitulate a number of functional deficits and pathological responses exhibited in human TBI cases. In this study, we employ a systems approach to improving the identification of biomarkers that can distinguish there two models. These biomarkers, if successfully identified, could be used to better guide treatments to mTBI patients, and more optimistically they could be potential targets of novel treatments.

Recent years have witnessed an increasing number of disease markers identified through computational analysis of genome-wide expression profiles. Typically, gene expression profiling studies are limited to focus on individual genes that are significantly differentially expressed between different classes of diseases. However, singlegene analyses have been criticized for several reasons $[10,11]$. In the cases of mTBI classification, if we only examined the differences in the expression levels of individual genes across different mTBI models and neglected the genes that are not associated with a TBI subtype at a significance threshold, we would fail to account for the complexities and redundancies that arise from gene interactions inherent to the mTBI responses. Discarded genes showing modest differential expression between mTBI classes may represent important biomarkers of mTBI. In this study, we have proposed a data-driven model and identified biomarkers not as individual genes but as gene subnetworks, by incorporating the gene expression profiles from injury models and the proteinprotein interaction information from existing databases. The genes in each of the identified subnetworks are expected to be highly correlated with each other and exhibit a coherent expression profile across samples, while others exist as background noise. It is also expected that the genes in a functional subnetwork exhibit high topological similarity with each other and should lead to a biologically meaningful sample classification. The network-based approach has been widely adopted to identify gene subnetworks as biomarkers in the field of cancer research and other human diseases [12, 13], but has never been applied in the discovery of biomarkers for brain injury. Here, with simulation and real data analysis, we have demonstrated that our computational systems approach based on network theory performs better than individual gene analyses as well as other gene grouping strategies in mTBI classification. The identified subnetworks can provide insights into the multifactorial relationships of genes and delineate the underlying complexities of the biological processes involved in different mTBI classes.

\section{Methods \\ Animal subjects and surgeries}

Male Sprague-Dawley (SD) rats (275-300 g) were purchased from Charles River Laboratories (Wilmington, MA). All experimental procedures were approved by the local Institutional Animal Care and Use Committee and were conducted according to the recommendations provided in the Guide for the Care and Use of Laboratory Animals. Protocols were designed to minimize pain and discomfort during the injury procedure and recovery period. Our $\mathrm{mCCI}$ and mFPI injury models were described in [3]. After injury preparation, animals were placed in a warm chamber and allowed to completely recover from anesthesia, and then returned to their home cages.

\section{Gene expression microarray}

Using the mTBI animals ( $n=4 /$ group), ipsilateral cortical issues underlying the injury site was quickly dissected at $24 \mathrm{~h}$ post-injury. Total RNA from the cortical tissue was isolated using the mirVana miRNA Isolation Kit (Invitrogen, Carlsbad, CA), following the manufacturers' recommended protocol, and amplified using the Illumina TotalPrep RNA Amplification Kit (Ambion, Austin, TX). RNA amplification and microarray hybridization were carried out by The University of Texas Health Science Center Houston Microarray Core Laboratory (Houston, TX). Briefly, first-strand complementary DNA (cDNA) was generated from total RNA by reverse transcription. Second strand cDNA synthesis was initiated by the addition of RNase H/DNA polymerase mix. The complementary RNA (cRNA) was amplified by the in vitro transcription reaction (IVT). cRNA (750 ng) was loaded onto RatRefSeq-12 Illumina Sentrix Beadchip Arrays (Illumina, Inc., San Diego, CA), hybridized overnight, washed, and incubated with streptavidin-Cy3 to detect hybridized biotin-labeled cRNA probes. Arrays were dried and scanned with a BeadArray Reader (Illumina). It was noted that most raw gene expression values were not normally distributed but highly skewed. Therefore, the Box-Cox transformation [14] was used to normalize the distribution for each gene expression values. The Kolmogorov-Smirnov test was used to test for normality of the transformed distribution at a 5\% significance level.

\section{Constructing a weighted network from protein interaction information and gene expression data}

Experimentally detected protein-protein interactions (PPIs) were downloaded from BioGRID [15], DIP [16], and HPRD [17] databases. Since there are a limited number of experiments detecting PPIs in the rat genome, we also obtained predicted rat PPIs based on onthology, 
where orthologous interactions were generated by mapping experimentally detected PPIs in human or mouse genomes to pairs of orthologs in rat genome, if such orthologs are available in HomoloGene database [18]. Each edge of the protein interaction network was further weighted by overlaying gene expression information. Specifically, we calculated the absolute value of the Pearson correlation coefficient $\operatorname{abs}\left(\operatorname{cor}\left(\mathrm{x}_{\mathrm{i}}, \mathrm{x}_{\mathrm{j}}\right)\right)$ as the edge weight, where $x_{i}$ and $x_{j}$ represented the normalized gene expression vectors for genes $i$ and $j$, respectively. Therefore, each edge of the protein interaction network was weighted by the level of co-expression between its two corresponding genes, with the weights between 0 and 1 .

\section{Identifying significant subnetworks}

We performed functional network analysis by following the protocol described in [19]. We defined the subnetwork scoring function $S$ as a weighted sum of class relevance $R$ and modularity $M$.

$$
\mathrm{S}=\beta \mathrm{M}+\mathrm{R}
$$

Here $M$ describes the subnetwork connectivity, and $R$ is a measure of the discriminatory power of the subnetwork genes to differentiate classes. In addition, the parameter $\beta$ allows us to trade off the effects of the gene expression information with the network modularity on the subnetwork score. To simplify the scoring algorithm, we set $\beta=1$, assuming equal weights of network modularity and class relevance on calculating the network score.

To get a measure of how strongly the genes within a subnetwork are connected, the modularity $M$ was calculated as the mean clustering coefficients $C_{i}$ of the genes in a subnetwork,

$$
\mathrm{M}=\frac{\sum_{\mathrm{i}} \mathrm{C}_{\mathrm{i}}}{\mathrm{n}}[\text { for } \mathrm{n}>=3 ; 0 \text { otherwise }]
$$

(3)| where $C_{i}$ was defined as in Dong and Horvath [20, 21]. $C_{i}$ is the clustering coefficient for node $i$, where nodes $l$ and $m$ are node neighbors of $i$, and $w$ represents the weight of the edge between nodes in the subnetwork:

$$
C_{i}=\frac{\sum_{l \neq i} \sum_{m \neq i, m \neq l} w_{i l} w_{l m} w_{m i}}{\left(\sum_{l \neq i} w_{i l}\right)^{2}-\sum_{l \neq i} w_{i l}^{2}}
$$

Intuitively, $C_{i}$ is a ratio of the weighted triangles that can be made with node $i$ and its neighbors over the sum of the weighted possible triangles extending off of node $i$. For a general weighted network with edge weights between 0 and 1 , the clustering coefficient of node $i, C_{i}$ also lies between 0 and $1 . C_{i}$ equals 1 if and only if all neighbors of node $i$ are connected to each other.

The class relevance $R$ is a measure of the ability for a subnetwork to distinguish two classes. To calculate this, the expression values of each gene $i$ in sample $j$ were first normalized to $\mathrm{z}$-scores, $\mathrm{z}_{\mathrm{ij}}$, which had a mean of 0 and standard deviation of 1 for each gene over all samples. The individual z-scores of each member gene in the subnetwork $K$ for sample $j$ were averaged into a summarized expression $\mathrm{Vkj}=\frac{\sum_{i=1}^{n} z_{i j}}{n}$, where $n$ is the number of genes in the subnetwork K. A t-test was then used to compare the summarized expression values of samples between two classes and the resulting t-value was denoted as the class relevance $R$. In this study, the two classes refer to $\mathrm{mCCI}$ and $\mathrm{mFPI}$ injury models.

A framework demonstrating the steps for finding significant subnetworks is described in Fig. 1. We applied the greedy search algorithm in searching subnetworks [22]. To identify significant subnetworks that discriminate $\mathrm{mCCI}$ and $\mathrm{mFPI}$, candidate subnetworks were scored comparing two classes. First, individual differentially expressed genes were used as seeds for growing potential subnetworks. For each seed, two neighboring genes were iteratively added to the seed and subnetwork scores were recalculated. The pair of neighboring genes that yielded the biggest improvement in subnetwork score was added to the seed to form an initial subnetwork of three genes (i.e., an initial triangular subnetwork). Single neighbor nodes were then added iteratively until the subnetwork score could no longer be improved. It is likely that genes are shared across different subnetworks, resulting in potentially redundant subnetworks. The redundant subnetworks were removed by the following steps:

1. Obtaining the scores of all subnetworks and sorting them in a descending order of scores.

2. Iterating through the list of subnetworks and checking for redundancy.

a) if a subnetwork was contained within a higherscoring subnetwork, we discarded the lower-scoring subnetwork;

b) if a subnetwork was a super set of a higher-scoring subnetwork, we discarded the super set;

c) if there was an overlap in genes between a lowerscoring and higher-scoring subnetwork:

i. if the overlap $\geq 50 \%$ (number of overlapping genes/ total number of unique genes), we discarded lowerscoring subnetwork;

ii. if the overlap $<50 \%$, we kept both subnetworks (document them for manual inspection).

To select the significant subnetworks, we calculated the empirical $p$-values of the identified subnetworks. We first generated the null distribution by permuting the expression vector of genes in the full network. This permutation test dissociated the relationship between protein interaction and 


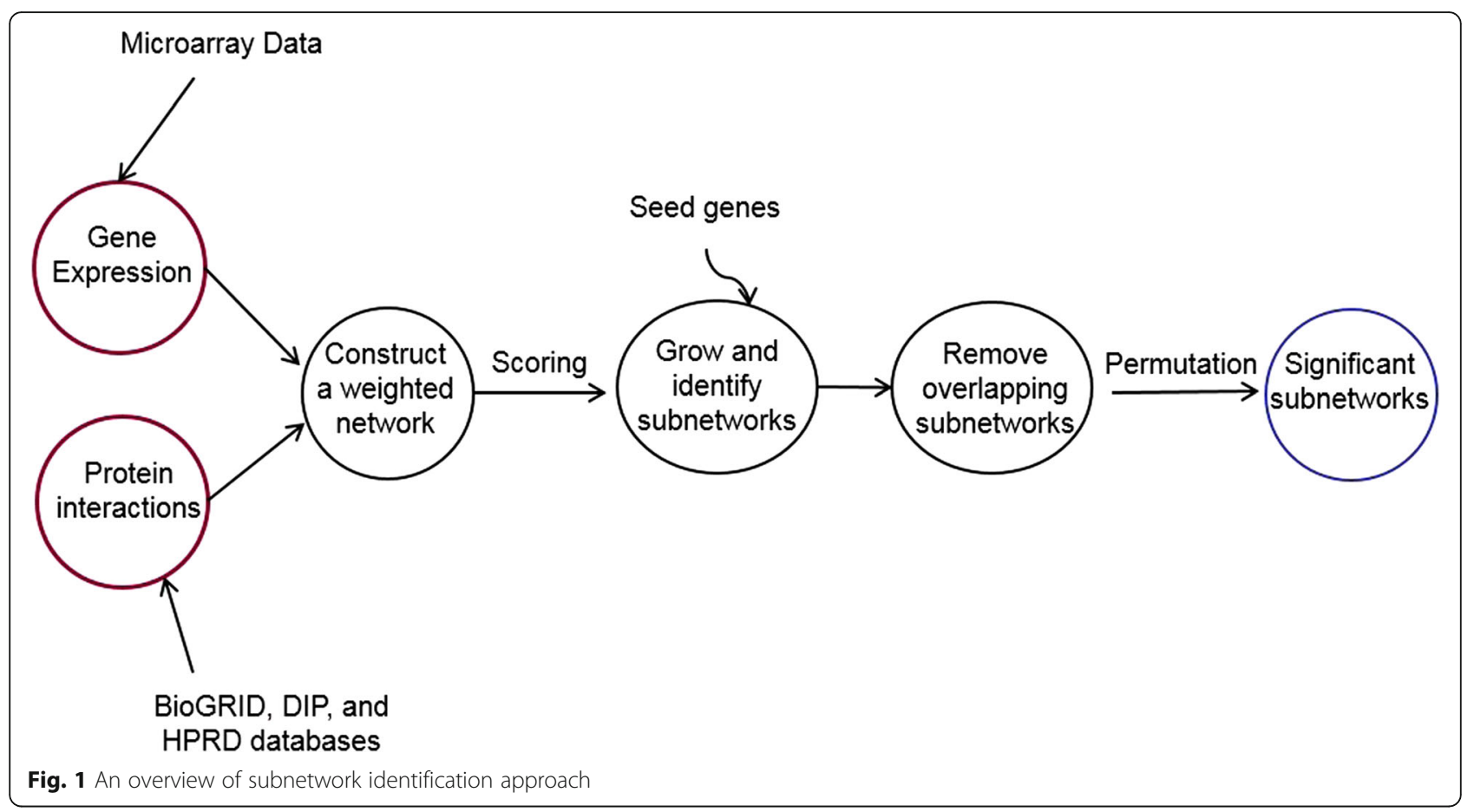

gene expression information. We then ran the same subnetwork identification procedure on the permuted data. This process was repeated 100 times and the scores of the resulted random subnetworks were recorded for each permutation. The empirical adjusted $p$-value for the real subnetwork score was calculated as the fraction of the random subnetworks having a higher score than that real subnetwork [23]. Only the subnetworks with empirical adjusted $p$-values smaller than 0.05 were selected for further evaluation and analysis.

\section{Gene grouping strategies}

To evaluate our approach for identifying biomarkers that distinguish different mTBI classes (mCCI vs. mFPI), we compared our approach with other gene grouping strategies to be used in classification. Two other gene grouping strategies were included in this study: 1) Pathway based gene sets using the list of canonical pathways extracted from the Molecular Signature Database (MSigDB) [24]. 2) Functionally related gene sets based on Gene Ontology (GO) annotations. GO gene sets were determined by retrieving genes for all GO terms that contained less than 50 genes, in order to eliminate the GO terms that were too general in function annotation. The resulted two groups of gene sets were evaluated for their discriminatory potential in classifying TBI classes. For each gene grouping strategy, expression values for each gene set were converted into summarized expression scores as described previously. These expression scores were used to test differential expression between mCCI and mFPI classes and gene sets were ranked according to their discriminatory powers. After redundant gene sets were removed, the resulting gene sets were used as features in training algorithms to build models for predicting TBI classes.

\section{Results}

Protein interaction network weighted with gene co-expression data

Given the resources of protein interactions as described in the Materials and Methods section, a protein interaction network was constructed with 18,781 proteins and 207,829 edges. Gene expression values were then overlaid on the protein interaction network. Each edge of this subnetwork was weighted by the level of co-expression between its two corresponding genes using Pearson correlation, as described previously. Fig. 2 shows the distribution of node degrees, weighted node degrees and edge weights for this network. There were 3 outlier gene nodes that had over 200 direct neighbors. These genes can be problematic depending on how subnetworks are searched for. In our current method of finding subnetworks, we aimed to find complex interactions within a small set of genes, more specifically, we looked for enriched triangular relationships amongst genes. Since these particular hub genes could generate a lot of star-shaped structures for subnetworks, they were removed from the network subject to further network analysis.

\section{Overview of functional subnetworks}

The subnetwork identification step yielded a total of 189 significant subnetworks, consisting of 695 genes. An example of the resulting discriminative subnetworks is shown in Fig. 3. The genes mitogen-activated-protein 

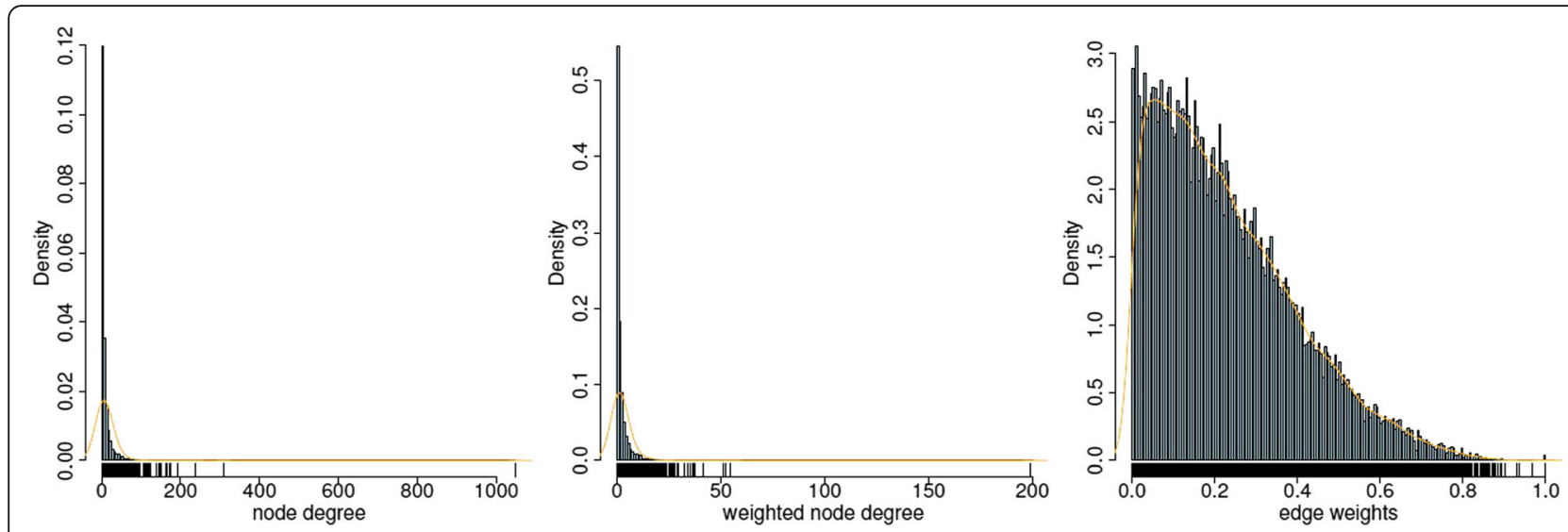

Fig. 2 Network structure profile. Distribution of node degrees in PPI, weighted node degrees and edge weights in the constructed weighted network. The $y$-axis represents the probability density for each of the distribution

kinase 1 (MAPK1) and interleukin 6 family cytokine (LIF) did not show significant differentiated expression between $\mathrm{mCCI}$ and mFPI samples, but they played an important role in the subnetwork by interconnecting many differentially expressed genes, such as CEBPB, MYC, JAK2 and STAT3. Given the fact that both MPAK1 and LIF genes are well-known players in the cytokine signaling pathway involved in inflammatory response, our results suggest they can serve as potential targets for intervention. To further investigate the functions of the identified subnetworks, we extracted the Biological Process annotations from Gene Ontology
(GO) database [25], and examined whether any GO terms were overrepresented by the union of genes in the 50 most significant subnetworks, compared to an expected genome-wide representation [26]. Because there is a lot of redundancy in the GO tree, we used the GO terms from levels 3 to 10 to determine specific biological process categories which the subnetwork genes belong to [27]. The GO enrichment $p$-values were calculated by the hyper-geometric test, followed by Benjamini and Hochberg's multiple hypotheses testing correction procedure [28]. The ten most significantly enriched GO terms are listed in Table 1. Overall, the GO analysis of

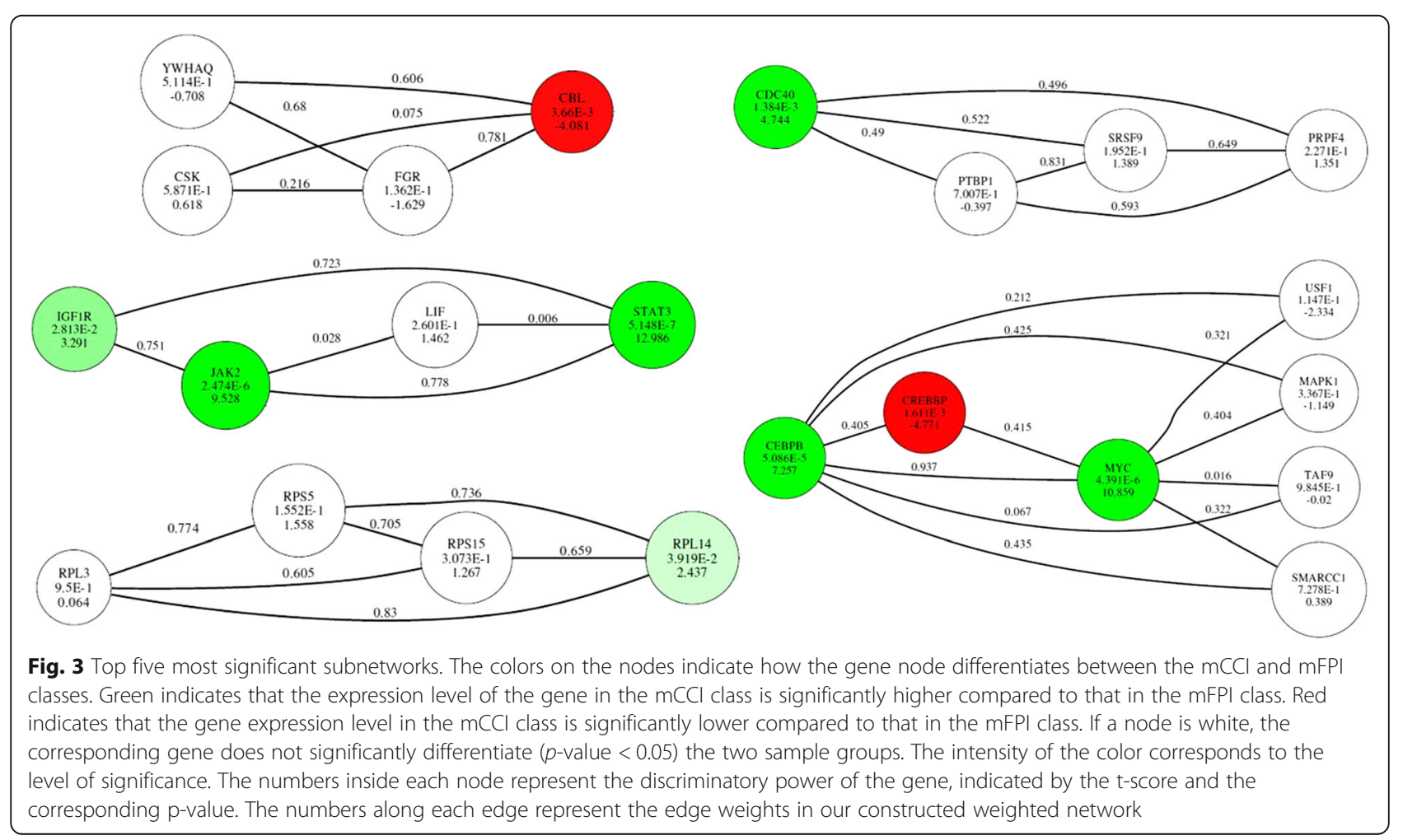


Table 1 Gene Ontology (GO) biological process annotations for significant subnetwork genes

\begin{tabular}{ll}
\hline GO Biological Process & FDR \\
\hline Dendrite development & $8.16 \mathrm{E}-08$ \\
Neuron development & $2.86 \mathrm{E}-07$ \\
Regulation of cell differentiation & $8.11 \mathrm{E}-07$ \\
Neurogenesis & $5.41 \mathrm{E}-06$ \\
Regulation of programmed cell death & $8.19 \mathrm{E}-06$ \\
Regulation of membrane potential & $1.02 \mathrm{E}-05$ \\
lon transmembrane transport & $1.13 \mathrm{E}-05$ \\
Cell-cell adhesion & $1.68 \mathrm{E}-05$ \\
Blood coagulation & $4.52 \mathrm{E}-05$ \\
Wnt signaling pathway & $6.32 \mathrm{E}-04$ \\
\hline
\end{tabular}

The top 10 most enriched GO biological process terms with their corresponding corrected $p$-values are listed. FDR, adjusted $p$-values for multiple testing by Benjamini and Hochberg's procedure

the top 50 subnetworks showed significant overrepresentation of genes belonging to some fundamental cellular processes, such as cell differentiation and cell-cell adhesion. We also found there were significant enrichment of GO terms related to brain injury, such as neuron development, neurogenesis, ion membrane transport, and blood coagulation.

\section{mTBI subtype classification evaluation}

Given the identified subnetworks, we tested their validity and performance in the classification problem. However, in this study, we only had experimental data available for 8 rats (4 samples/injury model). The small sample size made it difficult to train and test a classifier. Therefore, we performed a simulation study to achieve an unbiased classification evaluation. The mean and standard deviation of each gene were estimated from the observed data. Given these parameters, we used the packages in $\mathrm{R}$ studio [29] to simulate gene expression datasets corresponding to mCCI and mFPI classes, with 100 samples per class. Using the simulated datasets, we performed a five-fold cross validation to compute the classification accuracy. First, the simulated gene expression data corresponding to the identified subnetwork markers were used to encode features for a Support Vector Machine (SVM) classifier [30]. Then, we divided the mCCI and mFPI samples into five equal parts, respectively. We used four-fifth of the samples to train SVM and the remaining one-fifth of samples to test the trained classifier. Finally, we evaluated the sensitivity and specificity of our method and calculated its ROC curves and the areas under ROC curve (AUC). We compared the performance of TBI subtype classification of our subnetwork markers with the genes that were most significantly differentially expressed between $\mathrm{mCCI}$ and mFPI samples. We selected the top 695 individual genes, the same number of genes as that in the union of identified subnetworks, to achieve a fair comparison. Our subnetworks yielded an AUC score of 0.71 , while the individual gene set yielded a lower AUC score of 0.58 (Fig. 4). Therefore, the subnetwork markers identified based on our network analysis outperformed the individual gene markers in classifying mild TBI subtypes. In addition, we compared our method with another subnetwork identification method by Chuang et al., which didn't consider the subnetwork modularity when identifying subnetworks [13]. As a result, it led to many star-shaped networks. When comparing the method performance using ROC curves, we found our method performed better than the method by Chuang et al. [13], based on the comparison of sensitivity and specificity (Fig. 4).

We also compared the performance of TBI subtype classification of the subnetwork markers with those based on predefined functionally related genes extracted from GO annotation and canonical pathways. The classification results were evaluated using an F-score as in [31], where $\mathrm{F}=2^{*}$ Precision*Recall/(Precision + Recall). In this particular classification task, the precision is the proportion of classified mCCI samples that are true mCCIs, and the recall is the proportion of true $\mathrm{mCCI}$ samples that are correctly classified by a method. Similarly, we performed a five-fold cross validation to compute the classification accuracy. Using the features drawn from different gene sets, we trained SVM based on the simulated gene expression data from four-fifth of samples, and then we tested the performance of the learned feature weights on the remaining one-fifth of

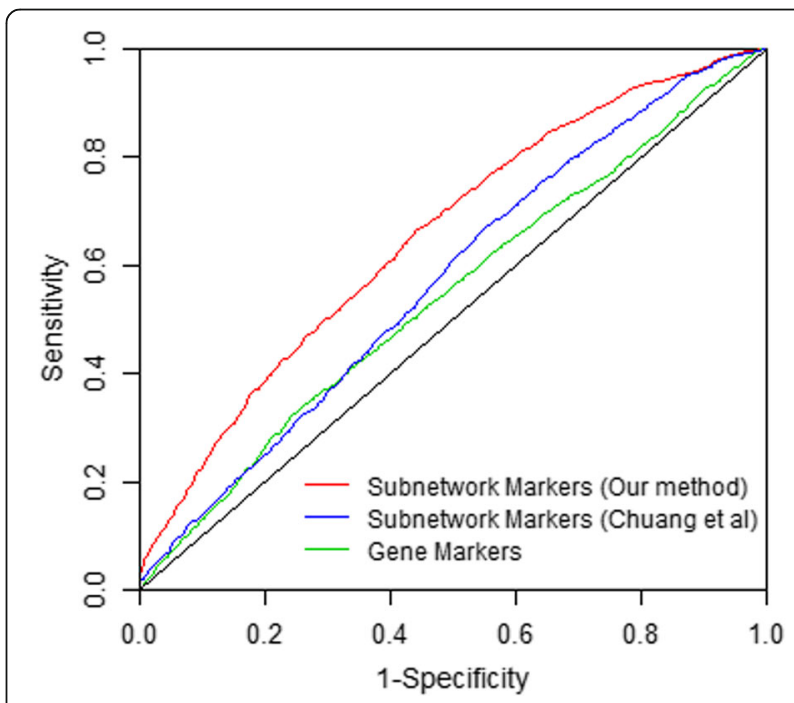

Fig. 4 Comparison of classification performance between subnetwork markers and individual genes. The activities of identified subnetworks, calculated as the mean activities of its member genes, are used as the features of the support vector machine (SVM) to classify the samples. The classifier performance is measured by ROC curves 


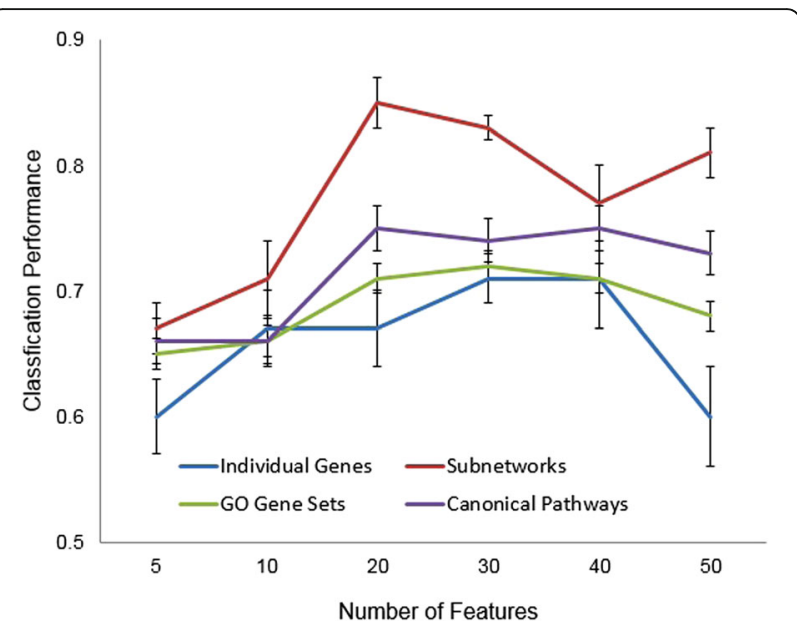

Fig. 5 Comparison of classification performance among subnetworks, individual genes, and predefined functionally gene sets from $\mathrm{GO}$ or MSigDB. For each size of feature set, five iterations of five-fold cross validation are used to split the dataset, train, and evaluate classifier. The curves show the median of classification performance, measured by the F-scores, and error bars indicate the standard deviation over five cross-validation experiments

samples. We repeated the process for five times to obtain an averaged F-score over iterations of cross validation experiments. We examined the classification accuracy using different sizes of feature set (the top 5 , $10,20,30,40$, or 50 features), and summarized the comparison results in Fig. 5. It is demonstrated that over all the tests, the SVM using 20 functional subnetwork features achieves the highest performance with an F-score of 0.85 . We have also shown the functional subnetworks outperform significant individual genes or predefined functionally related genes across different sizes of feature sets, indicating the advantage of using gene subnetworks for sample classification and prediction.

\section{Discussion}

The parameter setting in variable selection methods can impact the performance of the selected feature genes sets. In this study, we showed the relative robustness and superior performance of our network analysis across different numbers of selected features. We have demonstrated that effectively incorporating gene expression profiles into protein interaction information can identify functional subnetworks that better classify different classes of MTBI than the gene markers selected without network information. We understand that translating the knowledge gained from an animal model onto molecular biomarkers identification in patients is practically challenging, simply because the brain tissue in TBI patients is rarely available, but the use of peripheral tissues such as lymphoblast or blood could be a potential solution.

\section{Conclusions}

We have aimed to improve the identification of biomarkers that can distinguish two different classes of TBI in rodent animal models: the mild Controlled Cortical Impact (mCCI) and the mild Fluid Percussion Injury (mFPI), representing focal and diffuse TBIs, respectively. We have developed and applied a network-based approach on gene expression profiles from the entire rat genome. Our network based analysis can identify genes that are essential for maintaining the integrity of a subnetwork whose overall expression is discriminative between samples. In addition, we demonstrated that our network-based analysis achieves higher sensitivity and specificity in differentiating the heterogeneous responses corresponding to different classes of mTBI, compared to conventional analyses using either individual genes or predefined functionally related gene sets. These identified biomarkers could be used to better direct the diagnosis and treatment to TBI patients, and more optimistically, they could help to develop rationale-based therapies for treating the millions of Americans who suffer from TBI.

\section{Abbreviations \\ AUC: The areas under a receiver operating characteristic curve; GO: Gene ontology; KEGG: Kyoto encyclopedia of genes and genomes; mCCl: Mild controlled cortical impact; $\mathrm{mFPI}$ : Mild fluid percussion injury; \\ MSigDB: Molecular signature database; PPI: Protein-protein interaction; ROC curve: Receiver operating characteristic curve; SVM: Support vector machine; TBI: Traumatic brain injury}

\section{Acknowledgements}

Not applicable.

\section{Funding}

This study was supported in part by NIH grants R01 LM010022 and R01 CA163481.Publication charges for this article have been funded by the catalyst grant from the University of Texas Health Science Center at Houston. The funder had no role in the study design, data collection and analysis, decision to publish, or preparation of the manuscript.

Availability of data and materials

The source code and the dataset used for the current study is available upon request.

\section{About this supplement \\ This article has been published as part of BMC Systems Biology Volume 12 Supplement 8, 2018: Selected articles from the International Conference on Intelligent Biology and Medicine (ICIBM) 2018: systems biology. The full contents of the supplement are available online at https://bmcsystbiol.biomedcentral.com/ articles/supplements/volume-12-supplement-8.}

\section{Authors' contributions}

DP and YL conceived and designed the study. FASL performed the data analysis and drafted the manuscript. JR performed the animal experiments and joined the part of data analysis. YL coordinated the study and helped to draft the manuscript. All authors read and approved the final manuscript.

\section{Ethics approval}

All experimental procedures involving rats are approved by the local institutional animal care and use committee (IACUC). 


\section{Competing interests}

The authors declare that they have no competing interests.

\section{Publisher's Note}

Springer Nature remains neutral with regard to jurisdictional claims in published maps and institutional affiliations.

\section{Author details}

'Department of Epidemiology, University of Texas M.D. Anderson Cancer Center, 1155 Pressler Street, Houston, TX, USA. ${ }^{2}$ Department of Neurobiology and Anatomy, University of Texas Health Science Center at Houston, 6431 Fannin Street, Houston, TX, USA. ${ }^{3}$ University of Texas Graduate School of Biomedical Science, 6767 Bertner Avenue, Houston, TX, USA. ${ }^{4}$ Center for Precision Health, School of Biomedical Informatics, The University of Texas Health Science Center at Houston, 7000 Fannin Street, Houston, TX, USA.

\section{Published: 21 December 2018}

\section{References}

1. Yu Z, Morrison B 3rd. Experimental mild traumatic brain injury induces functional alteration of the developing hippocampus. J Neurophysiol. 2010; 103(1):499-510.

2. Grandhi R, Bonfield CM, Newman WC, Okonkwo DO. Surgical management of traumatic brain injury: a review of guidelines, pathophysiology, neurophysiology, outcomes, and controversies. J Neurosurg Sci. 2014:58(4): 249-59

3. Redell JB, Moore AN, Grill RJ, Johnson D, Zhao J, Liu Y, Dash PK. Analysis of functional pathways altered after mild traumatic brain injury. J Neurotrauma. 2013:30(9):752-64.

4. Albert-Weissenberger C, Siren AL. Experimental traumatic brain injury. Exp Transl Stroke Med. 2010;2(1):16.

5. Jeter CB, Hergenroeder GW, Hylin MJ, Redell JB, Moore AN, Dash PK. Biomarkers for the diagnosis and prognosis of mild traumatic brain injury/ concussion. J Neurotrauma. 2013;30(8):657-70.

6. Mychasiuk R, Farran A, Esser MJ. Assessment of an experimental rodent model of pediatric mild traumatic brain injury. J Neurotrauma. 2014;31(8): 749-57.

7. Jager TE, Weiss HB, Coben JH, Pepe PE. Traumatic brain injuries evaluated in $\mathrm{U}$. S. emergency departments, 1992-1994. Acad Emerg Med. 2000;7(2):134-40.

8. Vanderploeg RD, Curtiss G, Luis CA, Salazar AM. Long-term morbidities following self-reported mild traumatic brain injury. J Clin Exp Neuropsychol. 2007;29(6):585-98.

9. Saatman KE, Duhaime AC, Bullock R, Maas Al, Valadka A, Manley GT, Workshop Scientific T, Advisory Panel M. Classification of traumatic brain injury for targeted therapies. J Neurotrauma. 2008;25(7):719-38.

10. Fortney K, Kotlyar M, Jurisica I. Inferring the functions of longevity genes with modular subnetwork biomarkers of Caenorhabditis elegans aging. Genome Biol. 2010;11(2):R13.

11. Savarraj JPJ, Parsha K, Hergenroeder GW, Zhu L, Bajgur SS, Ahn S, Lee K, Chang T, Kim DH, Liu Y, et al. Systematic model of peripheral inflammation after subarachnoid hemorrhage. Neurology. 2017;88(16):1535-45.

12. Barabasi AL, Gulbahce N, Loscalzo J. Network medicine: a network-based approach to human disease. Nat Rev Genet. 2011;12(1):56-68.

13. Chuang HY, Lee E, Liu YT, Lee D, Ideker T. Network-based classification of breast cancer metastasis. Mol Syst Biol. 2007;3:140.

14. Sakia R. The box-cox transformation technique: a review. Statistician. 1992; 41:169-78

15. Chatr-Aryamontri A, Breitkreutz BJ, Oughtred R, Boucher L, Heinicke S, Chen D, Stark C, Breitkreutz A, Kolas N, O'Donnell L, et al. The BioGRID interaction database: 2015 update. Nucleic Acids Res. 2015:43(Database issue):D470-8.

16. Salwinski L, Miller CS, Smith AJ, Pettit FK, Bowie JU, Eisenberg D. The database of interacting proteins: 2004 update. Nucleic Acids Res. 2004, 32(Database issue):D449-51.

17. Goel R, Harsha HC, Pandey A, Prasad TS. Human protein reference database and human Proteinpedia as resources for phosphoproteome analysis. Mol BioSyst. 2012;8(2):453-63.

18. NCBI_Resource_Coordinators. Database resources of the National Center for biotechnology information. Nucleic Acids Res. 2016;44(D1):D7-19.

19. San Lucas A, Redell J, Dash P, Liu Y. Computer-assisted approaches to identify functional gene networks involved in traumatic brain injury. In:
Srivastava AK, Cox CS, editors. Pre-clinical and clinical methods in brain trauma research. New York, NY: Springer New York; 2018. p. 349-60.

20. Zhang B, Horvath S. A general framework for weighted gene co-expression network analysis. Stat Appl Genet Mol Biol. 2005:4:Article17.

21. Dong J, Horvath S. Understanding network concepts in modules. BMC Syst Biol. 2007;1:24.

22. Carey VJ, Gentry J, Whalen E, Gentleman R. Network structures and algorithms in Bioconductor. Bioinformatics. 2005;21(1):135-6.

23. Sham PC, Purcell SM. Statistical power and significance testing in large-scale genetic studies. Nat Rev Genet. 2014;15(5):335-46.

24. Liberzon A, Subramanian A, Pinchback R, Thorvaldsdottir H, Tamayo P, Mesirov JP. Molecular signatures database (MSigDB) 3.0. Bioinformatics. 2011;27(12):1739-40.

25. Huntley RP, Sawford T, Mutowo-Meullenet P, Shypitsyna A, Bonilla C, Martin MJ, O'Donovan C. The GOA database: gene ontology annotation updates for 2015. Nucleic Acids Res. 2015;43(Database issue):D1057-63.

26. Wang $Z, X u W$, Liu Y. Integrating full spectrum of sequence features into predicting functional microRNA-mRNA interactions. Bioinformatics. 2015; 31(21):3529-36

27. Mi H, Huang X, Muruganujan A, Tang H, Mills C, Kang D, Thomas PD. PANTHER version 11: expanded annotation data from gene ontology and Reactome pathways, and data analysis tool enhancements. Nucleic Acids Res. 2017;45(D1):D183-9.

28. Benjamini Y, Hochberg Y. Controlling the false discovery rate: a practical and powerfful approach to multiple testing. JRStatist SoC B. 1995;57(1):289-300.

29. Gentleman RC, Carey VJ, Bates DM, Bolstad B, Dettling M, Dudoit S, Ellis B, Gautier L, Ge Y, Gentry J, et al. Bioconductor: open software development for computational biology and bioinformatics. Genome Biol. 2004;5(10):R80.

30. Zhu Y, Shen X, Pan W. Network-based support vector machine for classification of microarray samples. BMC Bioinformatics. 2009;10(Suppl 1):S21.

31. Wang Z, San Lucas FA, Qiu P, Liu Y. Improving the sensitivity of sample clustering by leveraging gene co-expression networks in variable selection. BMC Bioinformatics. 2014;15:153.

Ready to submit your research? Choose BMC and benefit from:

- fast, convenient online submission

- thorough peer review by experienced researchers in your field

- rapid publication on acceptance

- support for research data, including large and complex data types

- gold Open Access which fosters wider collaboration and increased citations

- maximum visibility for your research: over $100 \mathrm{M}$ website views per year

At $\mathrm{BMC}$, research is always in progress.

Learn more biomedcentral.com/submissions 